\title{
Association Between Early Initiation of Breastfeeding and Reduced Risk of Respiratory Infection: Implications for Non-separation of Infant and Mother in the COVID-19 Context
}

Bindi Borg ( $\square$ bindi_borg@yahoo.com.au )

Macquarie University Faculty of Medicine and Health Sciences: Macquarie University Faculty of Medicine Health and Human Sciences https://orcid.org/0000-0002-2764-9788

Karleen Gribble

Western Sydney University

Karan Courtney Haag

UNICEF

Kedar Raj Parajuli

Government of Nepal Ministry of Health and Population

Seema Mihrshahi

The University of Sydney School of Public Health

\section{Research}

Keywords: early initiation of breastfeeding, acute respiratory infection (ARI), non-separation of mother and baby, maternal proximity, COVID-19 pandemic, SARS-CoV-2

Posted Date: November 30th, 2021

DOI: https://doi.org/10.21203/rs.3.rs-1071412/v1

License: (c) (i) This work is licensed under a Creative Commons Attribution 4.0 International License. Read Full License 


\section{Abstract}

Early initiation of breastfeeding, within one hour of birth, is vital for the health of newborns, and reduces morbidity and mortality. Secondary analysis of the 2016 Nepal Demographic and Health Survey (DHS) showed that early initiation of breastfeeding significantly reduced the risk of acute respiratory infection (ARI) in children under two years. Early initiation of breastfeeding requires maternal proximity. However, during the COVID-19 pandemic, guidance varied, with some recommending that infants and mothers with SARS-CoV-2 be isolated from one another. Nepal's Ministry of Health and Population recommended nonseparation, but the adherence to this guidance was inconsistent. Separation of infant and mother inhibits early initiation of breastfeeding and increases the risk that infants will suffer from ARIs. Maternal proximity, non-separation and early initiation of breastfeeding should be promoted in all birthing facilities.

\section{Background}

Initiation of breastfeeding within one hour of birth is vital for the health of newborns. It has been consistently shown that infants who do not breastfeed within the first hour of life are at elevated risk of neonatal mortality $[1,2]$. Apart from the immunological and nutritional value of breastfeeding for the infant, early breastfeeding initiation is also important for mothers, particularly in reducing the risk of excessive postpartum haemorrhage [3].

Early initiation of breastfeeding is especially critical in low- and middle-income countries, and forms part of the World Health Organization's Essential Newborn Care Practices [4]. In Nepal, the Ministry of Health and Population has strongly supported early initiation of breastfeeding for at least two decades. Yet up to half of all infants are not facilitated to breastfeed immediately and the rate of early initiation of breastfeeding is actually decreasing $[5,6]$.

Early initiation of breastfeeding requires uninterrupted contact, preferably skin-to-skin, between infants and their mothers [7]. However, during the COVID-19 pandemic, some government and non-government health authorities have recommended separation at birth of infants from mothers who were SARS-CoV-2 positive or suspected to be so [8]. These recommendations prevented early initiation of breastfeeding. Moreover, they were in opposition to a precautionary approach that would have prioritised maternal-infant proximity until such time as it was demonstrated that SARS-CoV-2 posed a greater risk than maternal separation [9].

Given the level of concern about the nature of SARS-CoV-2 respiratory virus and the potential risk posed to infant health, we conducted a secondary analysis of the Nepal Demographic and Health Survey 2016 (DHS) dataset to determine the impact of breastfeeding practices on the risk of acute respiratory infection (ARI) in children. Our analysis demonstrated that delayed initiation of breastfeeding was associated with an increased risk of ARI in Nepali children under two years of age. This suggests that in the current pandemic, ensuring that infants and mothers are kept together to facilitate the early initiation of breastfeeding is even more critical in order to protect infants and young children from SARS-CoV-2. 


\section{Secondary Analysis Method And Results}

We conducted a secondary analysis of the Nepal DHS 2016 data and found a robust relationship between initiation of breastfeeding within the first hour of birth (early initiation) and reduced risk of ARI in the previous two weeks in children under two years.

An initial comparison between the prevalence of ARI in children aged 0-23 months with whom breastfeeding had or had not been initiated early was performed using a chi-square test. Prevalence of ARI was lower in children with whom breastfeeding had been initiated early $(2.5 \%)$ compared to children with whom breastfeeding had not been initiated early (4.1\%).

A mixed-effects logistic regression was performed to explore the relationship further. In unadjusted analysis, the odds of children with whom breastfeeding had been initiated early having had an ARI in the past two weeks were lower and approaching significance, as shown in Table 1. When the analysis was adjusted for child, child and maternal, and child, maternal and household covariates respectively, the odds were significantly reduced $(\mathrm{OR}=0.53, \mathrm{Cl}=0.310 .94, \mathrm{p}=0.028 ; \mathrm{OR}=0.45, \mathrm{Cl}=0.23-0.90, \mathrm{p}=0.023$; $\mathrm{OR}=0.46, \mathrm{Cl}=0.23-0.92, \mathrm{p}=0.028$ respectively). The relationship remained robust at all levels of the model, which suggests a true association.

Table 1

Odds ratios of children with whom breastfeeding had been initiated early having had an ARI in the past 2 weeks $(n=1,968)$

\begin{tabular}{|ll|}
\hline & $\begin{array}{l}\text { OR (95\% Cl, p } \\
\text { value) }\end{array}$ \\
\hline Unadjusted & $\begin{array}{l}0.61(0.37-1.01, \\
0.053)\end{array}$ \\
\hline Adjusted for covariates related to: & \\
\hline child (sex, age, birth weight) & $0.53(0.31-0.94$, \\
& $0.028)$ \\
\hline child and mother (sex, age, birth weight, mother's education, mother's work) & $0.45(0.23-0.90$, \\
& $0.023)$ \\
\hline $\begin{array}{l}\text { child, mother and household (sex, age, birth weight, mother's education, mother's } \\
\text { work, caste, residence, wealth) }\end{array}$ & $\begin{array}{l}0.46(0.23-0.92, \\
0.028)\end{array}$ \\
\hline
\end{tabular}

\section{Discussion}

\section{Early initiation of breastfeeding and reduced risk of morbidity and mortality}

Our analysis shows a clear association between delayed initiation of breastfeeding and increased risk of ARI in Nepali children under two years of age. This concurs broadly with the existing literature on 
breastfeeding initiation, morbidity, and mortality.

The literature on early initiation of breastfeeding has tended to focus on delayed breastfeeding initiation and increased risk of all-cause mortality. One systematic review and meta-analysis showed that delaying breastfeeding initiation beyond the first hour after birth doubled the risk of all-cause neonatal mortality [1]. Another systematic review and meta-analysis including 136,047 subjects from birth to 12 months found that infants who initiate breastfeeding between 2-23 hours after birth are at a 33\% greater risk of all-cause mortality, compared to infants who initiate breastfeeding within an hour [2]. Initiation of breastfeeding more than 24 hours after birth is associated with a two-fold risk of all-cause neonatal mortality $[2,10]$. Early initiation of breastfeeding is especially important for preterm and low birthweight infants [11].

In Nepal, neonatal mortality (from 0-28 days) is currently 16 deaths per 1,000 live births and accounts for $57 \%$ under-five mortality in Nepal [6]. The 2030 SDG target for Nepal is 12 deaths per 1,000 live births and Nepal's Every Newborn Action Plan target is 11 deaths per 1000 live births by 2035 [12]. A study in southern Nepal showed that $19 \%$ of all neonatal deaths could be averted with early initiation of breastfeeding and that if breastfeeding was delayed for 24 hours or more, neonatal mortality increased 1.4 times [13].

Evidence on delayed breastfeeding initiation and morbidity is limited, and very few studies have explored the association between early initiation of breastfeeding and ARI risk. Hajeebhoy et al. found that prelacteal feeding and delayed breastfeeding initiation were associated with higher risk of ARI [14], which concurs with our finding that children who initiated breastfeeding more than an hour after birth doubled their risk of ARI. Another study showed that delayed initiation resulted in a significantly increased risk in several respiratory symptoms including (cough and difficulty breathing) in infants up to twelve months [15]. To our knowledge, ours is one of the first studies to explore the relationship between delayed breastfeeding initiation and ARI morbidity, and the first such study in Nepal.

\section{Biological basis}

There are biologically plausible mechanisms which may explain the relationship between early initiation of breastfeeding and reduced morbidity and mortality related to ARI. Early breastfeeding initiation ensures that the infant receives colostrum, which is rich in immune factors and promotes resistance to and recovery from infection [16]. It also tends to reduce the risk of hypothermia, which has been shown to contribute to neonatal morbidity and mortality [2], including in Nepal [17]. Furthermore, recent studies have demonstrated that breastmilk from mothers who have been infected with or vaccinated against SARS-CoV-2 contain antibodies against the virus which suggests that breastfeeding could protect infants from SARS-CoV-2 $[18,19]$. This concurs with studies showing that vaccinating mothers against influenza confers protection to their breastfeeding infants [20].

Other pathways are yet to be explored. For example the priming effect of early initiation of breastfeeding on the digestive system is well known [21], and there is some evidence that breastfeeding may have a 
similar protective effective on the microbiome of the upper respiratory tract [22-24]. This possible relationship between the respiratory microbiome and early initiation of breastfeeding that might explain the reduced risk of ARI and may have implications for protecting infants and young children from SARSCoV-2. Another explanation may be the impact of bioactive components on mucosa. It is well known that secretory immunoglobulin A and macroglobulins along with other bioactive factors such as oligosaccharides and lactoferrin in breastmilk protect intestinal mucosa and inhibit attachment of pathogens $[14,16,25]$. It is conceivable that breastmilk also coats the nasopharyngeal mucosa, thereby protecting against the transmission of SARS-CoV-2 and other bacteria and viruses responsible for respiratory illness $[20,26]$.

Early initiation of breastfeeding also increases the probability of exclusive breastfeeding, reduces the risk that infants will be given other foods and liquids from birth to six months and increases the likelihood of continued breastfeeding. Thus, early initiation of breastfeeding could contribute to protection from infection in the longer term [27, 28], all of which reduce the risk of ARI infection [14, 29].

\section{Breastfeeding initiation requires maternal proximity}

The most effective way to facilitate early initiation of breastfeeding is through placing infants prone, skinto-skin on their mother's chest immediately after birth. Healthy infants placed skin-to-skin are able to orient themselves towards the breast, crawl to the breast, self-attach and suckle [30]. The process of working towards and achieving breastfeeding after being placed skin-to-skin can take some time and so it is critical that skin-to-skin contact is not terminated before this occurs [7, 31]. Infants placed skin-to-skin have more effective first breastfeeds [27]. These breastfeeds occur during a time when infants have high levels of catecholamines (adrenaline and noradrenaline) in their system which may enhance learning [7]. Breastfeeds during skin-to-skin in the first hour of life form a foundation for continued effective feeding [7]. Infants placed skin-to-skin after birth are less likely to be exposed to infant formula in hospital and to breastfeed for longer than infants not placed skin-to-skin [27]. Skin-to-skin contact thus facilitates early initiation of breastfeeding, exclusivity of breastfeeding, and duration of breastfeeding. The converse is true where skin-to-skin contact and early initiation of breastfeeding are eschewed. Separating infants and mothers make early initiation of breastfeeding more difficult.

\section{The impact of the pandemic on uninterrupted contact, skin- to-skin and early initiation of breastfeeding}

An analysis of COVID-19 maternal and newborn care guidance from 33 countries found that only $21 \%$ recommended that infants of mothers with SARS-CoV-2 initiate breastfeeding within an hour of birth and only $27 \%$ recommended that infants of mothers with confirmed SARS-CoV-2 be placed skin-to-skin with their mothers after birth. Further, $27 \%$ of guidance documents actually recommended that infants of mothers with SARS-CoV-2 be isolated from one another [8]. Interrupting contact, especially skin-to-skin, between the infant and mother inhibits early initiation of breastfeeding and increases the risk that infants will suffer from ARIs.

\section{The Nepal context prior to the COVID-19 pandemic}


In Nepal, the 2004 National Neonatal Health Strategy [32], the 2016 Every Newborn Action Plan [33] and the 2019 Safe Motherhood and Newborn Health Road Map 2030 [12] all state that breastfeeding should be initiated within an hour of birth. The 2016 DHS reported that $55 \%$ of children under two years had initiated breastfeeding within an hour of birth [5]. Just three years later, the Nepal Multiple Indicator Cluster Survey (MICS) reported that early initiation of breastfeeding had decreased to $42 \%$ [6]. Granted, the early initiation of breastfeeding indicator is based on historical recall of what happened up to two years before the survey interview, and the surveys are different. However, the sampling and indicator are sufficiently comparable to demonstrate that early initiation of breastfeeding in Nepal, which was already inadequate, is not improving.

Unpublished data from Nepal's Health Information Management System (HMIS) [34] reports an even lower rate of early initiation of breastfeeding. This may be more accurate than the DHS or MICS data since there is no recall bias. Over the past five years, early initiation of breastfeeding has declined from approximately $30 \%$ in $2017-2019$, to $17 \%$ in 2020 , to $15 \%$ in 2021 .

Even before the COVID-19 pandemic, there were missed opportunities for early initiation of breastfeeding. Although $78 \%$ of births took place in health facilities in 2019 , only $42 \%$ of infants breastfed within an hour of birth [6]. Anecdotally, health workers say that they are so busy that they are not able to support breastfeeding, including early initiation. The largest maternity hospital in Kathmandu has acknowledged this deficit and aims to address it through a lactation support initiative that educates staff, mothers, fathers, and extended family on the importance of breastfeeding. UNICEF is supporting this initiative and is also working to invigorate the Baby Friendly Hospital Initiative by improving policy implementation and monitoring.

\section{The guidance on infant-mother non-separation in Nepal in the COVID-19 context}

Nepal's Ministry of Health and Population recommended infants born to women with SARS-CoV-2 infection or symptoms should not be separated, but should have skin-to-skin contact, early initiation of breastfeeding, rooming-in and direct breastfeeding [35]. However, a director of a maternity hospital in Kathmandu stated that isolation of infants from mothers with SARS-CoV-2 symptoms was hospital policy [36]. A study of nine hospitals found that during lockdown (March to May 2020), early initiation of breastfeeding decreased by 3.5\% [37], although there were significant differences between hospitals [38].

\section{Conclusion And Recommendations}

This research contributes to the body of evidence which shows that early initiation of breastfeeding and uninterrupted, preferably skin-to-skin, contact between newborns and their mothers is an essential first step for future health. Pertaining, as it does, to early initiation of breastfeeding and protection from ARI, it is particularly relevant in the COVID-19 era. In the face of SARS-CoV-2, a respiratory disease, it is more important than ever to ensure that mothers and babies stay together and establish breastfeeding within 
the first hour of life. This underscores the vital importance of non-separation of infants and mothers, even where mothers are SARS-CoV-2 positive or suspected.

This analysis should be repeated elsewhere, particularly in the South Asia region, to reinforce the implementation of guidance of non-separation after birth and early initiation of breastfeeding, irrespective of the mother's SARS-CoV-2 status. In Nepal, early initiation of breastfeeding and uninterrupted, preferably skin-to-skin, contact should continue to be promoted in all birthing facilities.

\section{Declarations}

Ethics approval and consent to participate: Ethics approval was sought and received in the original data collection.

Consent for publication: Not applicable

Availability of data and materials: The data is publicly available on the DHS website.

Competing interests: None

Funding: Funding was received from the University of Sydney.

Authors' contributions: SM and BB conceptualised the study. BB conducted the statistical analysis with support from SM. BB drafted the initial version of the manuscript. SM and KG provided significant additional input. $\mathrm{KCH}$ and KRP provided input and comments. All authors and approved the final manuscript.

Acknowledgements: None

\section{References}

1. Khan, J., et al., Timing of Breastfeeding Initiation and Exclusivity of Breastfeeding During the First Month of Life: Effects on Neonatal Mortality and Morbidity-A Systematic Review and Metaanalysis. Maternal and Child Health Journal, 2015. 19(3): p. 468-479.

2. Smith, E.R., et al., Delayed breastfeeding initiation and infant survival: A systematic review and meta-analysis. PLOS ONE, 2017. 12(7): p. e0180722.

3. Sobhy SI, Mohame NA, The effect of early initiation of breast feeding on the amount of vaginal blood loss during the fourth stage of labor. The Journal of the Egyptian Public Health Association, 2004. 79(1-2): p. 1-12.

4. World Health Organization. Regional Office for Europe, Essential newborn care and breastfeeding : training modules. 2002, Copenhagen : WHO Regional Office for Europe. 
5. Ministry of Health and Population, Nepal; New ERA; and ICF, Nepal Demographic and Health Survey 2016. 2017, Ministry of Health and Population, Nepal: Kathmandu, Nepal.

6. Central Bureau of Statistics (CBS), Nepal Multiple Indicator Cluster Survey 2019, Survey Findings Report. 2020, Central Bureau of Statistics and UNICEF Nepal: Kathmandu, Nepal.

7. Bystrova, K., et al., Early Contact versus Separation: Effects on Mother-Infant Interaction One Year Later. Birth, 2009. 36(2): p. 97-109.

8. Vu Hoang D, C.J., Gribble K, Marinelli K, \& Mathisen R, Misalignment of global COVID-19 breastfeeding and newborn care guidelines with World Health Organization recommendations. BMJ Nutrition, Prevention and Health, 2020. 3(2): p. 339-350.

9. Gribble, K., et al., Mistakes from the HIV pandemic should inform the COVID-19 response for maternal and newborn care. International Breastfeeding Journal, 2020. 15(1): p. 67.

10. Debes, A.K., et al., Time to initiation of breastfeeding and neonatal mortality and morbidity: a systematic review. BMC Public Health, 2013. 13(3): p. S19.

11. Lutter, C., Early Initiation of Breastfeeding: the Key to Survival and Beyond. 2010, PAHO.

12. Family Welfare Division, Nepal Safe Motherhood and Newborn Health Road Map 2030, M.o.H.a. Population, Editor. 2019, Government of Nepal: Kathmandu, Nepal.

13. Mullany, L.C., et al., Breast-feeding patterns, time to initiation, and mortality risk among newborns in southern Nepal. The Journal of nutrition, 2008. 138(3): p. 599-603.

14. Hajeebhoy, N., et al., Suboptimal breastfeeding practices are associated with infant illness in Vietnam. International Breastfeeding Journal, 2014. 9(1): p. 12.

15. Smith, E.R., et al., Delayed Breastfeeding Initiation Is Associated with Infant Morbidity. The Journal of pediatrics, 2017. 191: p. 57-62.e2.

16. Ballard, O. and A.L. Morrow, Human milk composition: nutrients and bioactive factors. Pediatric clinics of North America, 2013. 60(1): p. 49-74.

17. Mullany, L.C., et al., Neonatal hypothermia and associated risk factors among newborns of southern Nepal. BMC Medicine, 2010. 8(1): p. 43.

18. Juncker, H.G., et al., Antibodies Against SARS-CoV-2 in Human Milk: Milk Conversion Rates in the Netherlands. Journal of Human Lactation, 2021: p. 08903344211018185.

19. Perl, S.H., et al., SARS-CoV-2-Specific Antibodies in Breast Milk After COVID-19 Vaccination of Breastfeeding Women. JAMA, 2021. 325(19): p. 2013-2014. 
20. Schlaudecker, E.P., et al., IgA and neutralizing antibodies to influenza a virus in human milk: a randomized trial of antenatal influenza immunization. PloS one, 2013. 8(8): p. e70867-e70867.

21. van den Elsen, L.W.J., et al., Shaping the Gut Microbiota by Breastfeeding: The Gateway to Allergy Prevention? Frontiers in pediatrics, 2019. 7: p. 47-47.

22. Biesbroek, G., et al., The Impact of Breastfeeding on Nasopharyngeal Microbial Communities in Infants. American Journal of Respiratory and Critical Care Medicine, 2014. 190(3): p. 298-308.

23. Castanys-Muñoz, E., M.J. Martin, and E. Vazquez, Building a Beneficial Microbiome from Birth. Advances in Nutrition, 2016. 7(2): p. 323-330.

24. Gensollen, T., et al., How colonization by microbiota in early life shapes the immune system. Science (New York, N.Y.), 2016. 352(6285): p. 539-544.

25. Thai, J.D. and K.E. Gregory, Bioactive Factors in Human Breast Milk Attenuate Intestinal Inflammation during Early Life. Nutrients, 2020. 12(2): p. 581.

26. Kilpatrick R.M. and H.C. Meissner, What you need to know to reduce the risk of respiratory virus spread. American Academy of Pediatrics News, 2020.

27. Moore, E.R., et al., Early skin-to-skin contact for mothers and their healthy newborn infants. The Cochrane database of systematic reviews, 2016. 11(11): p. CD003519-CD003519.

28. Permatasari, T.A.E. and A. Syafruddin, Early Initiation of Breastfeeding Related to Exclusive Breastfeeding and Breastfeeding Duration in Rural and Urban Areas in Subang, West Java, Indonesia. Journal of Health Research, 2016. 30(5): p. 337-345.

29. Lamberti, L.M., et al., Breastfeeding for reducing the risk of pneumonia morbidity and mortality in children under two: a systematic literature review and meta-analysis. BMC public health, 2013. 13 Suppl 3(Suppl 3): p. S18-S18.

30. Widström, A.-M., et al., Skin-to-skin contact the first hour after birth, underlying implications and clinical practice. Acta paediatrica (Oslo, Norway : 1992), 2019. 108(7): p. 1192-1204.

31. Widström, A.-M., et al., Newborn behaviour to locate the breast when skin-to-skin: a possible method for enabling early self-regulation. Acta Paediatrica, 2011. 100(1): p. 79-85.

32. Ministry of Health and Population, National Neonatal Health Strategy. 2004: Kathmandu, Nepal.

33. Ministry of Health and Population, Nepal's Every Newborn Action Plan. 2016, Ministry of Health and Population: Kathmandu, Nepal.

34. Health Services Department, Management Division, Ministry of Health and Population. Health Management Information System (HMIS). Available from: http://202.70.86.2/hmis/dhis-web- 
commons/security/login.action

35. Ministry of Health and Population, Interim Guidance for RMNCH services in COVID 19 Pandemic, F.W. Division, Editor., Department of Health Services: Nepal.

36. Shrestha, E. and T. Heaton, Like most vulnerable groups, soon-to-be and new mothers face an uncertain near future, in The Kathmandu Post. 2020: Kathmandu.

37. Ashish, K.C., et al., Effect of the COVID-19 pandemic response on intrapartum care, stillbirth, and neonatal mortality outcomes in Nepal: a prospective observational study. The Lancet global health, 2020. 8(10): p. e1273-e1281.

38. Ashish, K.C., et al., The perfect storm: Disruptions to institutional delivery care arising from the COVID-19 pandemic in Nepal. Journal of global health, 2021. 11: p. 05010-05010. 